\title{
Neuromuscular Responses of the Vastus Lateralis to Slow Walking With Vascular Restriction
}

\section{ROBERT CARDENAS, CHARITY CAVAZOS, JOSE A LEAL JR, YOSHIAKI SATO,} MICHAEL G BEMBEN, \& MURAT KARABULUT

University of Texas at Brownsville, Texas Southmost College, University of Oklahoma

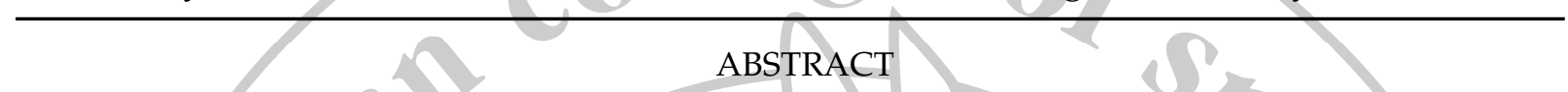

Motor unit recruitment of individual muscles could substantially differ with changes in speed and load. However, the recruitment patterns associated with low intensity walk training with vascular restriction at sustained loads and constant speed could be different than normal walking. Vascular restricted (VR) walk training is an effective method for increasing muscular strength; however the changes in motor unit recruitment and firing frequency have not been investigated to understand the mechanisms underlying the neuromuscular adaptations. Purpose: The purpose of this study was to analyze differences in electrical activity of the vastus lateralis during VR and non vascular restricted (non-VR) walking sessions. Methods: Seven healthy males [means $\pm(\mathrm{SD})$ : age $22.7 \pm(6.8)$ yrs, height $178 \pm(5.4) \mathrm{cm}$, weight $181.7 \pm(16.9)$ lbs.] performed both a VR walking session and a non-VR walking session for 20 minutes on a treadmill at a speed of $80.4 \mathrm{~m}-\mathrm{min}^{-1}$. During each walking period the subjects wore surface EMG electrodes that were placed along the longitudinal axis of the vastus lateralis (VL) of the right thigh at a distance of $33.3 \%$ between the lateral femoral epicondyle and the greater trochanter. A repeated measures ANOVA was used to determine the differences in both EMG amplitude (RMS) and median frequency of firing (MDF). Results: The VR walking session resulted in significantly greater RMS values compared to the non-VR walking session $(p<0.05)$; however there was no main effect for time or interaction between condition and time. For EMG MDF, there were no significant main effects for condition or time, and no significant interaction. Conclusion: The results indicate that walking during VR of the lower limbs may have a greater impact on neural adaptation of the VL due to increased motor unit activation as indicated by an increased RMS value. This might occur because blood flow restriction affects motor unit recruitment patterns resulting in the recruitment of more high-threshold, fast-twitch muscle fibers.

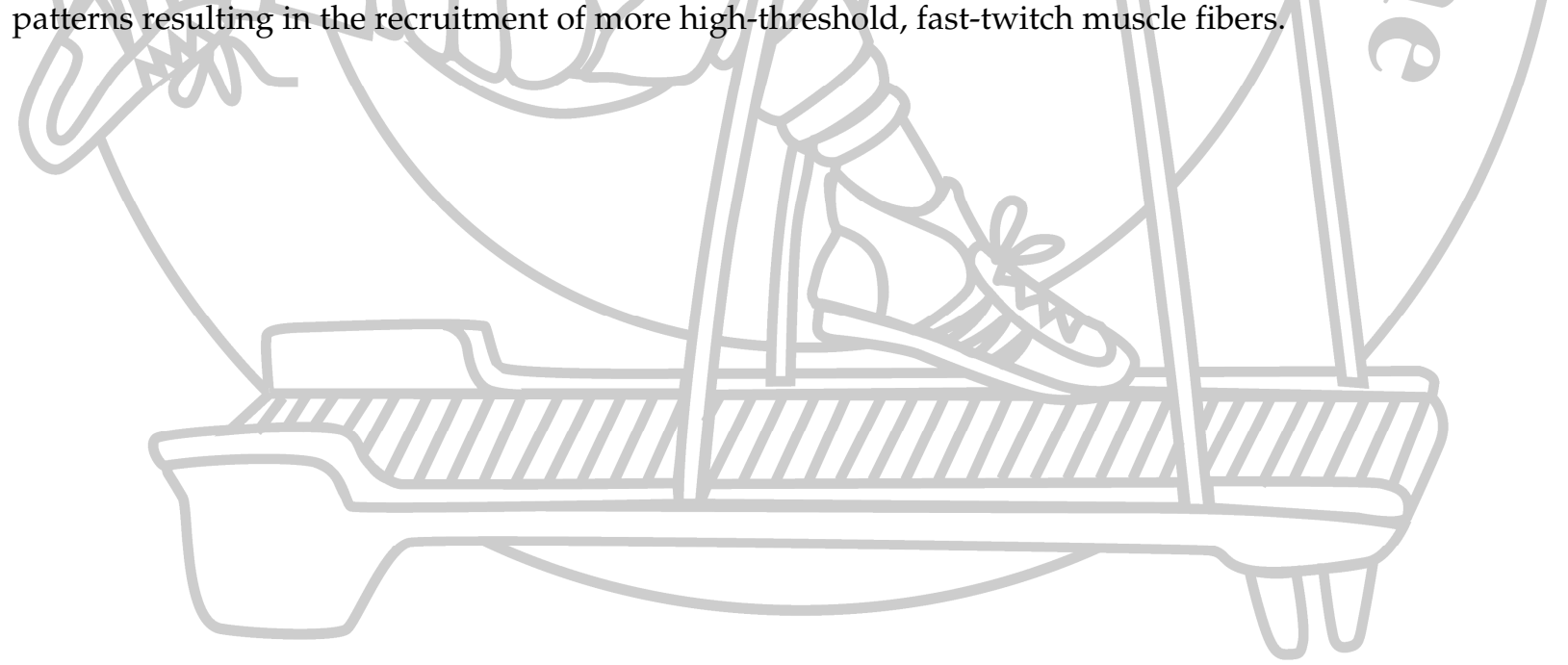

